\title{
THE SMOOTH-FIT PROPERTY IN AN EXPONENTIAL LÉVY MODEL
}

\author{
DAMIEN LAMBERTON, ${ }^{*}$ Université Paris-Est \\ MOHAMMED MIKOU, ${ }^{* *}$ Ecole Internationale des Sciences du Traitement \\ de l'Information
}

\begin{abstract}
We study the smooth-fit property of the American put price with finite maturity in an exponential Lévy model when the underlying stock pays dividends at a continuous rate. As in the perpetual case, a regularity property is sufficient for smooth fit to occur. We also derive conditions on the Lévy measure under which smooth fit fails.
\end{abstract}

Keywords: Optimal stopping; Lévy process; smooth-fit property; American option

2010 Mathematics Subject Classification: Primary 60G40; 60G51; 91G20

Secondary $60 \mathrm{~J} 75$

\section{Introduction}

The continuity of the derivative with respect to the underlying stock price of the American put price is a well-known property in the Black-Scholes model, called the smooth-fit property. In the context of exponential Lévy models, this property may no longer be true. Figure 1 demonstrates that in the CGMY model, one of the most used exponential Lévy models in practice (see [5]), the smooth-fit property holds when the parameter $Y=1$ and it fails when $Y=0.2$.

In the case of perpetual American options, a necessary and sufficient condition for smooth fit was derived by Alili and Kyprianou [1] in an exponential Lévy model without dividends. The picture is still unclear in the finite horizon case, except for the jump diffusion model (see [14]).

This paper deals with the smooth-fit property of the American put price in a general Lévy model when the underlying stock pays dividends at a continuous rate. We first show that the condition derived by Alili and Kyprianou is also sufficient for smooth fit in the finite horizon case (see Theorem 4.1). This condition is typically not satisfied when the logarithmic stock price is a Lévy process with finite variation and positive drift. In this case, we prove that, for large maturities, the smooth-fit property is not satisfied (see Theorem 4.3). Under a slightly stronger condition, we derive a lower bound for the jump of the derivative (see Theorem 4.2 and Remark 4.1). In the perpetual case, we also propose a proof of a slightly weaker version of Alili and Kyprianou's result, based on the variational inequality.

The paper is organized as follows. In Section 2 we describe the exponential Lévy model with dividends, and the basic properties of the perpetual and finite horizon American put prices in this model. Section 3 is devoted to the properties of the free boundary in the infinite and

Received 22 July 2010; revision received 13 September 2011.

* Postal address: Laboratoire d'Analyse et de Mathématiques Appliquées, Université Paris-Est, UMR CNRS 8050, 5 Boulevard Descartes, F-77454 Marne-la-Vallée Cedex 2, France. Email address: damien.lamberton@univ-mlv.fr

** Postal address: Laboratoire de Mathématiques, Ecole Internationale des Sciences du Traitement de l'Information, avenue du Parc, 95011 Cergy-Pontoise Cedex, France. Email address: mohammed.mikou@eisti.eu 

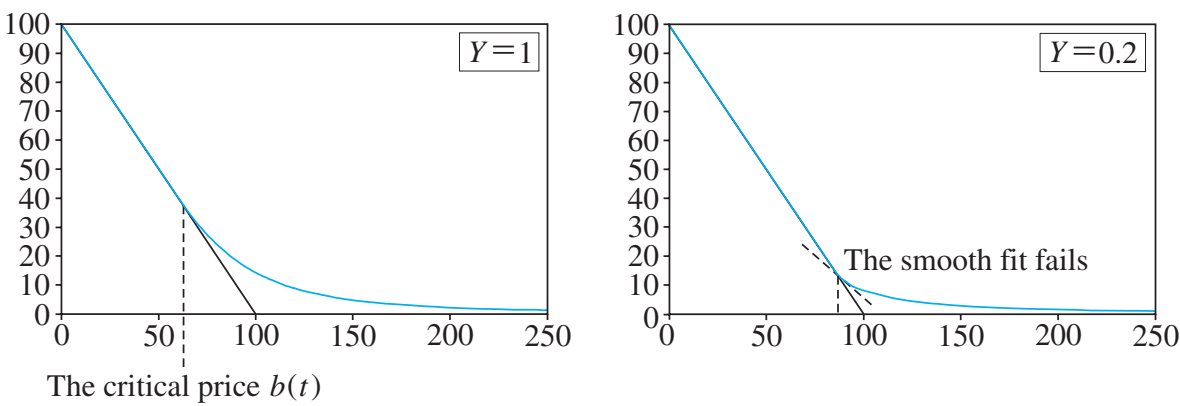

FIGURE 1: An example where the smooth fit fails in the CGMY model.

finite horizon cases. In Section 4 we study the smooth-fit property in the finite horizon case. In Section 5 we deal with the smooth-fit property in the perpetual case.

\section{The American put price in an exponential Lévy model}

\subsection{Lévy processes}

A real Lévy process $X$ is a real-valued stochastic process, starting from 0 , with stationary and independent increments. Without loss of generality, we may and will assume that the sample paths of $X$ are right continuous with left limits. The random process $X$ can be interpreted as the independent superposition of a Brownian motion with drift and an infinite superposition of independent (compensated) Poisson processes. More precisely, the Lévy-Itô decomposition (see [13]) gives the following representation of $X$ :

$$
\begin{aligned}
X_{t} & =\gamma t+\sigma B_{t}+Y_{t}, \\
Y_{t} & =\tilde{X}_{t}+\lim _{\varepsilon \rightarrow 0} \tilde{X}_{t}^{\varepsilon}, \\
\tilde{X}_{t} & =\int_{0}^{t} \int_{|x| \geq 1} x J_{X}(\mathrm{~d} s \times \mathrm{d} x), \\
\tilde{X}_{t}^{\varepsilon} & =\int_{0}^{t} \int_{\varepsilon \leq|x|<1} x \tilde{J}_{X}(\mathrm{~d} s \times \mathrm{d} x) .
\end{aligned}
$$

Here $\gamma$ and $\sigma$ are real numbers, $\left(B_{t}\right)_{t \geq 0}$ is a Brownian motion, $J_{X}$ is a Poisson measure on $\mathbb{R}_{+} \times(\mathbb{R} \backslash\{0\})$, and $\tilde{J}_{X}$ is the compensated Poisson measure $\tilde{J}_{X}(\mathrm{~d} t, \mathrm{~d} x)=J(\mathrm{~d} t, \mathrm{~d} x)-\mathrm{d} t v(\mathrm{~d} x)$. The measure $v$ is a positive Radon measure on $\mathbb{R} \backslash\{0\}$, called the Lévy measure of $X$, and it satisfies

$$
\int_{\mathbb{R}} 1 \wedge x^{2} v(\mathrm{~d} x)<\infty
$$

Note that the terms on the right-hand side of (2.1) are independent and the convergence of the last term is almost surely uniform with respect to $t$ on $[0, T]$. The Lévy-Itô decomposition entails that the distribution of $X$ is uniquely determined by $\left(\sigma^{2}, \gamma, v\right)$, called the characteristic triplet of the process $X$. The characteristic function of $X_{t}$ for $t \geq 0$ has the following Lévy-Khinchin representation (see [13]):

$$
\Phi_{X_{t}}(z)=\mathrm{E}\left(\mathrm{e}^{\mathrm{i} z X_{t}}\right)=\exp [t \phi(z)], \quad z \in \mathbb{C},
$$

with

$$
\phi(z)=-\frac{1}{2} \sigma^{2} z^{2}+\mathrm{i} \gamma z+\int\left(\mathrm{e}^{\mathrm{i} z x}-1-\mathrm{i} z x \mathbf{1}_{\{|x| \leq 1\}}\right) \nu(\mathrm{d} x) .
$$


The Lévy process $X$ is a Markov process and its infinitesimal generator is given by

$$
l f(x)=\frac{\sigma^{2}}{2} \frac{\partial^{2} f}{\partial x^{2}}(x)+\gamma \frac{\partial f}{\partial x}(x)+\int\left(f(x+y)-f(x)-y \frac{\partial f}{\partial x}(x) \mathbf{1}_{\{|y| \leq 1\}}\right) v(\mathrm{~d} y)
$$

for every $f \in \mathcal{C}_{b}^{2}(\mathbb{R})$, where $\mathcal{C}_{b}^{2}(\mathbb{R})$ denotes the set of all bounded, twice continuously differentiable functions with bounded derivatives. We complete this subsection by recalling two classical results about Lévy processes with finite variation (see [13]).

Proposition 2.1. A Lévy process is of finite variation if and only if its characteristic triplet $\left(\sigma^{2}, \gamma, v\right)$ satisfies

$$
\sigma=0 \text { and } \int_{|x| \leq 1}|x| v(\mathrm{~d} x)<\infty
$$

Remark 2.1. It follows from Proposition 2.1 that, for a finite-variation Lévy process with characteristic triplet $\left(\sigma^{2}, \gamma, v\right)$, we have

$$
\lim _{a \rightarrow 0} a v((-\infty,-a])=0 .
$$

Theorem 2.1. Let $X$ be a finite-variation Lévy process with characteristic triplet $(0, \gamma, v)$. We have

$$
\lim _{t \rightarrow 0^{+}} \frac{X_{t}}{t}=\gamma-\int_{|x| \leq 1} x v(\mathrm{~d} x) \text { almost surely (a.s.). }
$$

\subsection{The exponential Lévy model}

Let $\left(S_{t}\right)_{t \in[0, T]}$ be the price of a financial asset modeled as a stochastic process on a filtered probability space $\left(\Omega, \mathcal{F},\left(\mathcal{F}_{t}\right), \mathrm{P}_{0}\right)$. We suppose that there exists an equivalent (risk neutral) probability $\mathrm{P}$ under which the discounted underlying process is a martingale. In the exponential Lévy model, the risk neutral dynamics of $S_{t}$ under P are given by

$$
S_{t}=S_{0} \mathrm{e}^{(r-\delta) t+X_{t}},
$$

where the interest rate $r$ and the dividend rate $\delta$ are nonnegative constants, and $\left(X_{t}\right)_{t \in[0, T]}$ is a real Lévy process with characteristic triplet $\left(\sigma^{2}, \gamma, v\right)$. We include $r$ and $\delta$ in (2.4) for ease of notation.

Under $\mathrm{P}$, the discounted dividend adjusted stock price $\left(\mathrm{e}^{-(r-\delta) t} S_{t}\right)_{t \in[0, T]}$ is a martingale, which is equivalent to the following two conditions on the characteristic triplet (see [6, Proposition 3.17]):

$$
\int_{|x| \geq 1} \mathrm{e}^{x} v(\mathrm{~d} x)<\infty
$$

and

$$
\frac{\sigma^{2}}{2}+\gamma+\int\left(\mathrm{e}^{x}-1-x \mathbf{1}_{\{|x| \leq 1\}}\right) v(\mathrm{~d} x)=0 .
$$

We suppose that conditions (2.5) and (2.6) are satisfied in the sequel. We deduce from (2.5) that the infinitesimal generator defined in (2.2) can be written as

$$
l f(x)=\frac{\sigma^{2}}{2}\left(\frac{\partial^{2} f}{\partial x^{2}}-\frac{\partial f}{\partial x}\right)(x)+\int\left(f(x+y)-f(x)-\left(\mathrm{e}^{y}-1\right) \frac{\partial f}{\partial x}(x)\right) v(\mathrm{~d} y) .
$$


The stock price $\left(S_{t}\right)_{t \in[0, T]}$ is also a Markov process; we denote by $L$ its infinitesimal generator. From (2.7) we deduce that

$$
L f(x)=\frac{x^{2} \sigma^{2}}{2} \frac{\partial^{2} f}{\partial x^{2}}(x)+x(r-\delta) \frac{\partial f}{\partial x}(x)+\mathcal{B} f(x),
$$

where

$$
\mathscr{B} f(x)=\int v(\mathrm{~d} y)\left(f\left(x \mathrm{e}^{y}\right)-f(x)-x\left(\mathrm{e}^{y}-1\right) \frac{\partial f}{\partial x}(x)\right) .
$$

\subsection{The American put price}

In this model, the value at time $t$ of an American put with maturity $T$ and strike price $K$ is given by

$$
P_{t}=\operatorname{ess} \sup _{\tau \in \mathcal{T}_{t, T}} \mathrm{E}\left(\mathrm{e}^{-r \tau} \psi\left(S_{\tau}\right) \mid \mathcal{F}_{t}\right)
$$

where $\psi(x)=(K-x)_{+}$and $\mathcal{T}_{t, T}$ denotes the set of stopping times satisfying $t \leq \tau \leq T$. Owing to the Markov property (see [7] and [10]), we have

$$
P_{t}=P\left(t, S_{t}\right)
$$

where

$$
P(t, x)=\sup _{\tau \in \mathcal{T}_{0, T-t}} \mathrm{E}\left(\mathrm{e}^{-r \tau} \psi\left(S_{\tau}^{x}\right)\right)
$$

with $S_{t}^{x}=x \mathrm{e}^{(r-\delta) t+X_{t}}$. The following proposition follows easily from (2.9).

Proposition 2.2. For $t \in[0, T]$, the function $x \mapsto P(t, x)$ is nonincreasing and convex on $[0,+\infty)$.

For $x \in[0,+\infty)$, the function $t \mapsto P(t, x)$ is continuous and nonincreasing on $[0, T]$.

We recall the following proposition about the variational inequality related to the American put in the exponential Lévy model (see [9, Theorem 3.1]).

Theorem 2.2. The distribution $\left(\partial_{t}+L-r\right) P$ is a nonpositive measure on $(0, T) \times \mathbb{R}$, and, on the open set $\{(t, x) \in(0, T) \times \mathbb{R} \mid P(t, x)>\psi(x)\}$, we have $\left(\partial_{t}+L-r\right) P=0$.

\subsection{The perpetual American put price}

The perpetual American put price is an American put price with maturity $T$ equal to $\infty$. So, as previously, the value at time $t$ of a perpetual American put with strike price $K$ is given by

$$
P_{t}^{*}=\operatorname{ess} \sup _{\tau \in \mathcal{T}_{t, \infty}} \mathrm{E}\left(\mathrm{e}^{-r \tau} \psi\left(S_{\tau}\right) \mid \mathcal{F}_{t}\right)
$$

where $\psi(x)=(K-x)_{+}$and $\mathcal{T}_{t, \infty}$ denotes the set of stopping times satisfying $t \leq \tau$. Owing to the fact that the process $X$ has stationary and independent increments, it can be proved that

$$
P_{t}^{*}=P^{*}\left(S_{t}\right)
$$

where

$$
P^{*}(x)=\sup _{\tau \in \mathcal{T}_{0, \infty}} \mathrm{E}\left(\mathrm{e}^{-r \tau} \psi\left(S_{\tau}^{x}\right)\right),
$$

with $S_{t}^{x}=x \mathrm{e}^{(r-\delta) t+X_{t}}$. The following proposition follows easily from (2.10). 
Proposition 2.3. The function $x \mapsto P^{*}(x)$ is nonincreasing and convex on $[0,+\infty)$.

As in the finite horizon case, the perpetual American put in the exponential Lévy model satisfies the following variational inequality (see [9, Theorem 3.1]).

Theorem 2.3. The distribution $(L-r) P^{*}$ is a nonpositive measure on $\mathbb{R}^{+}$, and, on the open set $\left\{x \in \mathbb{R}^{+} \mid P^{*}(x)>\psi(x)\right\}$, we have $(L-r) P^{*}=0$.

\section{Properties of the free boundary}

\subsection{The finite horizon case}

Throughout this subsection, we will assume that at least one of the following conditions is satisfied:

$$
\sigma \neq 0, \quad v((-\infty, 0))>0, \quad \text { or } \quad \int_{(0,+\infty)}(x \wedge 1) v(\mathrm{~d} x)=+\infty .
$$

Under this assumption, we have (as observed in [9]), for all $t \in[0, T)$ and all $x \in[0,+\infty)$,

$$
P(t, x)>0 \text {. }
$$

We will also assume that $r>0$. The critical price at time $t \in[0, T)$ is defined by

$$
b(t)=\inf \{x \geq 0 \mid P(t, x)>\psi(x)\} .
$$

Note that, since $t \mapsto P(t, x)$ is nonincreasing, the function $t \mapsto b(t)$ is nondecreasing. It follows from (3.1) that $b(t) \in[0, K)$. We obviously have $P(t, x)=\psi(x)$ for $x \in[0, b(t))$ and also for $x=b(t)$, due to the continuity of $P$ and $\psi$. We also deduce from the convexity of $x \mapsto P(t, x)$ that, for all $t \in[0, T)$ and all $x>b(t)$,

$$
P(t, x)>\psi(x) .
$$

Then, the continuation region $C$ can be written as

$$
C=\{(t, x) \in[0, T) \times[0,+\infty) \mid x>b(t)\} .
$$

The graph of $b$ is called the exercise boundary or free boundary.

We recall the following properties of $t \mapsto b(t)$ (see [9, Section 4]).

Theorem 3.1. The function $t \mapsto b(t)$ is continuous and $b(t)>0$ on $[0, T)$.

We also recall from [9] the following result, which characterizes the limit of the critical price $b(t)$ as $t$ approaches $T$.

Theorem 3.2. If $\int\left(\mathrm{e}^{x}-1\right)_{+} v(\mathrm{~d} x) \leq r-\delta$, we have $\lim _{t \rightarrow T} b(t)=K$.

If $\int\left(\mathrm{e}^{x}-1\right)+v(\mathrm{~d} x)>r-\delta$, we have $\lim _{t \rightarrow T} b(t)=\xi$, where $\xi$ is the unique real number in the interval $(0, K)$ such that

$$
\varphi(\xi)=r K
$$

where $\varphi$ is the function defined by

$$
\varphi(x)=\bar{\varphi}(x)+\delta x
$$

and

$$
\bar{\varphi}(x)=\int\left(x \mathrm{e}^{y}-K\right)_{+} v(\mathrm{~d} y), \quad x \in(0, K) .
$$




\subsection{The perpetual case}

Assume that $P^{*}>0$. The critical price in this case is defined by

$$
b^{*}=\inf \left\{x \geq 0 \mid P^{*}(x)>\psi(x)\right\} .
$$

Note that, since $x \mapsto P^{*}(x)$ is a nonincreasing convex function, and $P^{*}>0$, we have $b^{*} \in(0, K)$ (one can prove that $b^{*}<K$ by the same argument as in [9, p. 574]). In this case, the continuation region $C^{*}$ can be written as

$$
C^{*}=\left\{x \in[0,+\infty) \mid x>b^{*}\right\}=\left(b^{*},+\infty\right) .
$$

\section{The smooth-fit principle in the finite horizon case}

Throughout this section, we will assume that $r>0$.

To a fixed level $x \in \mathbb{R}$, we associate the first strict passage time $\tau_{x}^{-}$below $x$ for the process $\left(\log \left(S_{t} / S_{0}\right)\right)$, i.e.

$$
\tau_{x}^{-}=\inf \left\{t \in(0, T) \mid(r-\delta) t+X_{t}<x\right\},
$$

with the convention that inf $\varnothing=T$. Recall that 0 is regular for $(-\infty, 0)$ if and only if $\mathrm{P}\left(\tau_{0}^{-}=0\right)=1$. Define

$$
d:=r-\delta-\int\left(\mathrm{e}^{x}-1\right) v(\mathrm{~d} x)
$$

Note that, if $X$ has finite variation, we have, from Theorem 2.1,

$$
\lim _{t \rightarrow 0} \frac{(r-\delta) t+X_{t}}{t}=d .
$$

So that $d$ appears as the drift of the logarithmic stock price. The following proposition is a summary of what is known from the literature (see [1, Proposition 7] or [8, Theorem 6.5]).

Proposition 4.1. The point 0 is regular for $(-\infty, 0)$ if and only if one of the following three conditions holds:

1. $X$ has finite variation and $d<0$,

2. $X$ has finite variation, $d=0$, and

$$
\int_{-1}^{0^{-}} \frac{|x| v(\mathrm{~d} x)}{\int_{0}^{|x|} v(y,+\infty) \mathrm{d} y}=+\infty,
$$

\section{X has infinite variation.}

The second case was added to the class of processes exhibiting regularity of 0 for the lower half-line in [4] and, for the other cases, we refer the reader to the discussion at the beginning of [3, Section VI.3].

The following theorem gives a sufficient condition for the smooth-fit property.

Theorem 4.1. If 0 is regular for $(-\infty, 0)$ then the smooth-fit principle is satisfied.

The proof of this result was given to us by G. Peskir. Note that the idea goes back to Bather [2] in the case of Brownian motion (see [12, Section 9.2]). In fact, it was conjectured in [1] that regularity is a necessary and sufficient condition for smooth fit in the case of strong Markov processes. This conjecture was disproved for diffusions in [11]. 
Proof of Theorem 4.1. Suppose that 0 is regular for $(-\infty, 0)$, and fix $t \in[0, T)$. We want to show that $x \mapsto P(t, x)$ is differentiable at $b(t)$ and that $\partial_{x} P(t, b(t))=\psi^{\prime}(b(t))$ (smooth fit), where $b(t) \in(0, K)$ is the critical price. To simplify the proof, we consider $t=0$. First note that, for $h>0$,

$$
\frac{P(0, b(0)+h)-P(0, b(0))}{h} \geq \frac{\psi(b(0)+h)-\psi(b(0))}{h},
$$

since $P \geq \psi$ and $P(t, b(0))=\psi(b(0))$. So, it follows that

$$
\liminf _{h \rightarrow 0^{+}}\left(\frac{P(0, b(0)+h)-P(0, b(0))}{h}\right) \geq \psi^{\prime}(b(0)) .
$$

Next we consider the optimal stopping time related to $P(0, b(0)+h)$, i.e.

$$
\begin{aligned}
\tau_{h} & =\inf \left\{t \in[0, T) \mid S_{t}^{b(0)+h}<b(t)\right\} \\
& =\inf \left\{t \in[0, T) \mid(r-\delta) t+X_{t} \leq \ln \left(\frac{b(t)}{b(0)+h}\right)\right\} \\
& \leq \inf \left\{t \in[0, T) \mid(r-\delta) t+X_{t} \leq \ln \left(\frac{b(0)}{b(0)+h}\right)\right\} \\
& =: \tau_{h}^{*},
\end{aligned}
$$

where the inequality follows from the fact that $(b(t))_{t \in[0, T)}$ is nondecreasing. Recall that $\mathrm{P}\left(\tau_{0}^{-}=0\right)=1$. On the set $\left\{\tau_{0}^{-}=0\right\}$, given a fixed $t \in(0, T)$, there exists $s \in[0, t]$ such that $(r-\delta) s+X_{s}<0$. For small enough $h$, we have $(r-\delta) s+X_{s}<\ln (b(0) /(b(0)+h))$, so that $\tau_{h}^{*} \leq s$. Therefore, $\lim _{h \rightarrow 0} \tau_{h}^{*} \leq t$. Since $t$ is arbitrary, we deduce that $\tau_{h}^{*} \rightarrow 0$ a.s. when $h$ goes to 0 . Hence,

$$
\lim _{h \rightarrow 0} \tau_{h}=0 \quad \text { a.s. }
$$

Moreover, since

$$
P(0, b(0)) \geq \mathrm{E}\left(\mathrm { e } ^ { - r \tau _ { h } } \psi \left(b(0) \mathrm{e}^{\left.\left.(r-\delta) \tau_{h}+X_{\tau_{h}}\right)\right),}\right.\right.
$$

we have

$$
\begin{aligned}
& \frac{P(0, b(0)+h)-P(0, b(0))}{h} \\
& \quad=\frac{\mathrm{E}\left(\mathrm { e } ^ { - r \tau _ { h } } \psi \left(b(0) \mathrm{e}^{\left.\left.(r-\delta) \tau_{h}+X_{\tau_{h}}\right)\right)-P(0, b(0))}\right.\right.}{h} \\
& \quad \leq \mathrm{E}\left(\mathrm{e}^{-r \tau_{h}} \frac{\psi\left((b(0)+h) \mathrm{e}^{(r-\delta) \tau_{h}+X_{\tau_{h}}}\right)-\psi\left(b(0) \mathrm{e}^{\left.(r-\delta) \tau_{h}+X_{\tau_{h}}\right)}\right.}{h}\right) .
\end{aligned}
$$

Since $\psi$ is continuously differentiable in a neighborhood of $b(0)$, we have

$$
\lim _{h \rightarrow 0} \frac{\psi\left((b(0)+h) \mathrm{e}^{\left.(r-\delta) \tau_{h}+X_{\tau_{h}}\right)-\psi\left(b(0) \mathrm{e}^{\left.(r-\delta) \tau_{h}+X_{\tau_{h}}\right)}\right.}\right.}{h}=\psi^{\prime}(b(0)) .
$$

Then, using the Lipschitz continuity of $\psi$, by dominated convergence we obtain

$$
\limsup _{h \rightarrow 0}\left(\frac{P(0, b(0)+h)-P(0, b(0))}{h}\right) \leq \psi^{\prime}(b(0)) .
$$

Combining (4.1) and (4.2), we deduce the theorem. 
It is well known that if $X$ has infinite variation, 0 is regular (see Theorem 4.1), so we have smooth fit. We will now assume that $X$ has finite variation. Define $d^{+}:=r-\delta-\int\left(\mathrm{e}^{x}-\right.$ $1)_{+} v(\mathrm{~d} x)$. Note that $d=d^{+}+\int\left(\mathrm{e}^{y}-1\right)_{-} v(\mathrm{~d} y)$. Recall that, if $d<0,0$ is regular for $(-\infty, 0)$, so the smooth-fit property is satisfied. We will prove below (see Theorem 4.3) that if $d>0$, the smooth-fit property cannot be satisfied, at least for large maturities. Under the stronger condition $d_{+} \geq 0$, we have a more precise result.

Theorem 4.2. If $X$ has finite variation and $d^{+} \geq 0$, we have

$$
\partial_{x}^{+} P(t, b(t)) \neq \partial_{x}^{-} P(t, b(t))
$$

for every $t \in(0, T)$.

Proof. Let $t \in[0, T)$ and $x \geq 0$, and suppose that $X$ is a finite-variation Lévy process such that $d^{+} \geq 0$. In this case, the infinitesimal generator in (2.8) can be written as

$$
L f(x)=x\left[r-\delta-\int\left(\mathrm{e}^{y}-1\right) v(\mathrm{~d} y)\right] \frac{\partial f}{\partial x}(x)+\int v(\mathrm{~d} y)\left[f\left(x \mathrm{e}^{y}\right)-f(x)\right]
$$

for all $f \in \mathcal{C}_{b}^{1}(\mathbb{R})$, where $\mathcal{C}_{b}^{1}(\mathbb{R})$ denotes the set of all bounded $\mathcal{C}^{1}$ functions with bounded derivative. Recall that, from Theorem 2.2, $\left(\partial_{t}+L-r\right) P=0$ in the sense of distributions on the continuation region $C$. So $(L-r) P \geq 0$ since $t \mapsto P(t, x)$ is nonincreasing. Also, $x \mapsto P(t, x)$ is convex, so its right derivative $\partial_{x}^{+} P$ is bounded and right continuous. Then, from (4.3) we deduce that

$$
\begin{aligned}
& b(t)\left[r-\delta-\int\left(\mathrm{e}^{y}-1\right) v(\mathrm{~d} y)\right] \partial_{x}^{+} P(t, b(t))+\int v(\mathrm{~d} y)\left[P\left(t, b(t) \mathrm{e}^{y}\right)-P(t, b(t))\right] \\
& \quad \geq r P(t, b(t)) .
\end{aligned}
$$

Note that $P(t, b(t))=\psi(b(t))=K-b(t), P\left(t, b(t) \mathrm{e}^{y}\right)=\psi\left(b(t) \mathrm{e}^{y}\right)=K-b(t) \mathrm{e}^{y}$ if $y<0$, and $P\left(t, b(t) \mathrm{e}^{y}\right) \leq P(t, b(t))$ if $y>0$. So, from (4.4) we obtain

$$
\begin{aligned}
b(t)[ & \left.r-\delta-\int\left(\mathrm{e}^{y}-1\right) v(\mathrm{~d} y)\right] \partial_{x}^{+} P(t, b(t)) \\
& \geq-\int_{y<0} v(\mathrm{~d} y)\left[\left(K-b(t) \mathrm{e}^{y}\right)-(K-b(t))\right]+r(K-b(t)) \\
& =b(t) \int_{y<0}\left(\mathrm{e}^{y}-1\right) v(\mathrm{~d} y)+r(K-b(t)) \\
& =-b(t) \int\left(\mathrm{e}^{y}-1\right)_{-} v(\mathrm{~d} y)+r(K-b(t)) .
\end{aligned}
$$

Note also that $d=d^{+}+\int\left(\mathrm{e}^{y}-1\right)_{-} v(\mathrm{~d} y)$. If we had $d=0$, we would deduce that $v(-\infty, 0)=$ 0 and (4.5) would become

$$
r(K-b(t)) \leq b(t) \int\left(\mathrm{e}^{y}-1\right)_{-} v(\mathrm{~d} y)=0,
$$

which is in contradiction with the facts that $r>0$ and $b(t) \in(0, K)$. Therefore, we must have $d>0$, and (4.5) now gives

$$
\partial_{x}^{+} P(t, b(t)) \geq \frac{-\int\left(\mathrm{e}^{y}-1\right)_{-} \nu(\mathrm{d} y)+r(K / b(t)-1)}{r-\delta-\int\left(\mathrm{e}^{y}-1\right)_{+} \nu(\mathrm{d} y)+\int\left(\mathrm{e}^{y}-1\right)_{-} \nu(\mathrm{d} y)}>-1 .
$$

We conclude the theorem since $\partial_{x}^{-} P(t, b(t))=\psi^{\prime}(b(t))=-1$. 
Remark 4.1. If $d^{+}>0$, we can see from (4.6) the following explicit lower bound for the jump of the derivative:

$$
\partial_{x}^{+} P(t, b(t))+1 \geq \frac{d^{+}}{d}>0
$$

for every $t \in[0, T]$.

We will now prove that if $d$ is positive, the smooth-fit property fails, at least for large values of the maturity.

Theorem 4.3. If $X$ is a finite-variation Lévy process and $d>0$, and if $T>K / d b^{*}$, where $b^{*}$ is the critical price of the perpetual put, there exists $t \in[0, T)$ such that

$$
\partial_{x}^{+} P(t, b(t))>-1 .
$$

We first show the following lemma.

Lemma 4.1. Assume that $X$ is a finite-variation Lévy process and $d>0$. Fix $t \in[0, T)$, and assume that $\partial_{+} P(t, b(t))=\partial_{-} P(t, b(t))$. Then, we have

$$
\limsup _{h \rightarrow 0} \frac{b(t+h)-b(t)}{h} \geq b^{*} d,
$$

where $b^{*}$ is the critical price of the perpetual put.

Proof. To simplify the proof, we consider the case $t=0$. Let $h>0$, and suppose that the smooth-fit property is satisfied at $t=0$. Let $\tau_{h}$ be the optimal stopping time related to $P(0, b(0)+h)$, i.e.

$$
\tau_{h}=\inf \left\{t \in[0, T) \mid S_{t}^{b(0)+h}<b(t)\right\}=\inf \left\{t \in[0, T) \mid(r-\delta) t+X_{t}<\ln \left(\frac{b(t)}{b(0)+h}\right)\right\},
$$

with the convention that inf $\varnothing=T$. Note that $\tau_{h}$ is nonnegative and nondecreasing with respect to $h$. We denote by $\tau_{0}$ the limit of $\tau_{h}$ when $h$ goes to 0 . Note also that by the zero-one law we have $\mathrm{P}\left(\tau_{0}=0\right) \in\{0,1\}$. We now discuss both cases.

Case 1: $\mathrm{P}\left(\tau_{0}=0\right)=0$. Note that $\tau_{0} \leq \tau_{h}$ and

$$
\begin{aligned}
P(0, b(0)+h) & =\mathrm{E}\left(\mathrm { e } ^ { - r \tau _ { h } } \psi \left((b(0)+h) \mathrm{e}^{\left.\left.(r-\delta) \tau_{h}+X_{\tau_{h}}\right)\right)}\right.\right. \\
& \geq \mathrm{E}\left(\mathrm { e } ^ { - r \tau _ { 0 } } \psi \left((b(0)+h) \mathrm{e}^{\left.\left.(r-\delta) \tau_{0}+X_{\tau_{0}}\right)\right) .}\right.\right.
\end{aligned}
$$

So, by letting $h$ go to 0 we have

$$
P(0, b(0))=\mathrm{E}\left(\mathrm { e } ^ { - r \tau _ { 0 } } \psi \left(b(0) \mathrm{e}^{\left.\left.(r-\delta) \tau_{0}+X_{\tau_{0}}\right)\right)} .\right.\right.
$$

Then, using the convexity of $\psi$, we obtain

$$
\begin{aligned}
& \frac{P(0, b(0)+h)-P(0, b(0))}{h} \\
& \quad \geq \mathrm{E}\left(\mathrm{e}^{-r \tau_{0}} \frac{\psi\left((b(0)+h) \mathrm{e}^{(r-\delta) \tau_{0}+X_{\tau_{0}}}\right)-\psi\left(b(0) \mathrm{e}^{\left.(r-\delta) \tau_{0}+X_{\tau_{0}}\right)}\right.}{h}\right) \\
& \quad \geq \mathrm{E}\left(\mathrm{e}^{-r \tau_{0}} \psi_{d}^{\prime}\left(b(0) \mathrm{e}^{(r-\delta) \tau_{0}+X_{\tau_{0}}}\right) \mathrm{e}^{\left.(r-\delta) \tau_{0}+X_{\tau_{0}}\right)}\right. \\
& \quad=-\mathrm{E}\left(\mathrm{e}^{-\delta \tau_{0}+X_{\tau_{0}}} \mathbf{1}_{\left\{(r-\delta) \tau_{0}+X_{\tau_{0}}<\ln (K / b(0))\right\}}\right) .
\end{aligned}
$$


Now, suppose that $\delta>0$. Since $\tau_{0}>0$ a.s. and $\mathrm{e}^{X}$ is a martingale, we obviously have

$$
\liminf _{h \rightarrow 0} \frac{P(0, b(0)+h)-P(0, b(0))}{h} \geq-\mathrm{E}\left(\mathrm{e}^{-\delta \tau_{0}+X_{\tau_{0}}}\right)=-\mathrm{E}\left(\mathrm{e}^{-\delta \tau_{0}}\right)>-1 .
$$

On the other hand, if $\delta=0$, (4.7) becomes

$$
\begin{aligned}
P(0, b(0)) & =\mathrm{E}\left(\mathrm { e } ^ { - r \tau _ { 0 } } \psi \left(b(0) \mathrm{e}^{\left.\left.r \tau_{0}+X_{\tau_{0}}\right)\right)}\right.\right. \\
& =K \mathrm{E}\left(\mathrm{e}^{-r \tau_{0}} \mathbf{1}_{\left\{r \tau_{0}+X_{\tau_{0}} \leq \ln (K / b(0))\right\}}\right)-b(0) \mathrm{E}\left(\mathrm{e}^{X_{\tau_{0}}} \mathbf{1}_{\left\{r \tau_{0}+X_{\tau_{0}} \leq \ln (K / b(0))\right\}}\right) .
\end{aligned}
$$

Since $P(0, b(0))=K-b(0)$, we derive

$$
K\left[1-\mathrm{E}\left(\mathrm{e}^{-r \tau_{0}} \mathbf{1}_{\left\{r \tau_{0}+X_{\tau_{0}} \leq \ln (K / b(0))\right\}}\right)\right]=b(0)\left[1-\mathrm{E}\left(\mathrm{e}^{X_{\tau_{0}}} \mathbf{1}_{\left.\left.\left\{r \tau_{0}+X_{\tau_{0}} \leq \ln (K / b(0))\right\}\right)\right] .} .\right.\right.
$$

Note that the left-hand side is positive because $\tau_{0}>0$ a.s. and $r>0$. Therefore,

$$
\mathrm{E}\left(\mathrm{e}^{X_{\tau_{0}}} \mathbf{1}_{\left\{r \tau_{0}+X_{\tau_{0}} \leq \ln (K / b(0))\right\}}\right)<1
$$

and (4.8) gives

$$
\liminf _{h \rightarrow 0} \frac{P(0, b(0)+h)-P(0, b(0))}{h} \geq-\mathrm{E}\left(\mathrm{e}^{X_{\tau_{0}}} \mathbf{1}_{\left\{r \tau_{0}+X_{\tau_{0}}<\ln (K / b(0))\right\}}\right)>-1 .
$$

We deduce from (4.9) and (4.10) that the smooth fit fails for every $\delta \geq 0$.

Case 2: $\mathrm{P}\left(\tau_{0}=0\right)=1$. We then have $\lim _{h \rightarrow 0} \tau_{h}=0$ a.s. In particular, $\tau_{h}<T$ for $h$ close to 0 and from the definition of $\tau_{h}$ we have

$$
(r-\delta) \tau_{h}+X_{\tau_{h}} \leq \ln \left(b\left(\tau_{h}\right)\right)-\ln (b(0)+h) \leq \ln \left(b\left(\tau_{h}\right)\right)-\ln (b(0)) .
$$

Therefore, using Theorem 2.1 and (2.6), we have

$$
\begin{aligned}
r-\delta+\lim _{h \rightarrow 0}\left(\frac{X_{\tau_{h}}}{\tau_{h}}\right) & =r-\delta+\gamma-\int_{\{|y| \leq 1\}} y \nu(\mathrm{d} y) \\
& =d \\
& \leq \liminf _{h \rightarrow 0} \frac{\ln \left(b\left(\tau_{h}\right)\right)-\ln (b(0))}{\tau_{h}} \\
& \leq \limsup _{t \rightarrow 0} \frac{\ln (b(t))-\ln (b(0))}{t} \\
& \leq \frac{1}{b(0)} \limsup _{t \rightarrow 0} \frac{b(t)-b(0)}{t} \\
& \leq \frac{1}{b^{*}} \limsup _{t \rightarrow 0} \frac{b(t)-b(0)}{t} .
\end{aligned}
$$

Hence,

$$
\limsup _{h \rightarrow 0} \frac{b(h)-b(0)}{h} \geq b^{*} d .
$$

Proof of Theorem 4.3. Suppose that the smooth fit is satisfied for every $t \in[0, T)$. Recall that $t \mapsto b(t)$ is a continuous nondecreasing function on $[0, T)$. So $b$ is almost everywhere (a.e.) differentiable on $[0, T)$ and from Lemma 4.1 we have

$$
b^{\prime}(t) \geq b^{*} d>0 \quad \text { a.e. on }[0, T) .
$$

Therefore, by integrating the last inequality, we obtain $K \geq b(t)-b(0) \geq d b^{*} t$. Finally, we get a contradiction for $T>K / d b^{*}$. 


\section{The perpetual put}

The following theorem can be proved by the same argument as in the finite horizon case.

Theorem 5.1. If 0 is regular for $(-\infty, 0)$ then the smooth-fit principle is satisfied.

We also have the following result, which has already been proved by Alili and Kyprianou [1]. Our contribution is only to give a proof based on the variational inequality.

Theorem 5.2. If $X$ has finite variation and $d>0$, the smooth-fit principle is not satisfied.

Proof. Suppose that the smooth-fit principle is satisfied and $d>0$. From Theorem 2.3 and (4.3) we have, for $x \geq b^{*}$,

$$
x\left(r-\delta-\int\left(\mathrm{e}^{y}-1\right) v(\mathrm{~d} y)\right) \partial_{x} P^{*}(x)+\int\left(P^{*}\left(x \mathrm{e}^{y}\right)-P^{*}(x)\right) v(\mathrm{~d} y)-r P^{*}(x)=0 .
$$

In particular, for $x=b^{*}$, we deduce, from $\partial_{x} P^{*}\left(b^{*}\right)=-1$ and $P^{*}\left(b^{*}\right)=K-b^{*}$, that

$$
b^{*} \delta+\int\left(P^{*}\left(b^{*} \mathrm{e}^{y}\right)-\left(K-b^{*} \mathrm{e}^{y}\right)\right) v(\mathrm{~d} y)=r K .
$$

Note that (5.1) can be written as

$$
x \delta+x d\left(\partial_{x} P^{*}(x)+1\right)+\int\left(P^{*}\left(x \mathrm{e}^{y}\right)-P^{*}(x)+x\left(\mathrm{e}^{y}-1\right)\right) v(\mathrm{~d} y)=r\left(P^{*}(x)+x\right) .
$$

Subtracting (5.2) from (5.3), we obtain

$$
\begin{aligned}
& \left(x-b^{*}\right) \delta+x d\left(\partial_{x} P^{*}(x)+1\right) \\
& +\int\left(P^{*}\left(x \mathrm{e}^{y}\right)-P^{*}(x)+x\left(\mathrm{e}^{y}-1\right)-\left(P^{*}\left(b^{*} \mathrm{e}^{y}\right)-\left(K-b^{*} \mathrm{e}^{y}\right)\right)\right) v(\mathrm{~d} y) \\
& \quad=r\left(P^{*}(x)-(K-x)\right) .
\end{aligned}
$$

For $y \in \mathbb{R}$, let $f_{y}$ be the function defined by $f_{y}(x)=P^{*}\left(x \mathrm{e}^{y}\right)-P^{*}(x)+x\left(\mathrm{e}^{y}-1\right)$. Then (5.4) becomes

$$
\left(x-b^{*}\right) \delta+x d\left(\partial_{x} P^{*}(x)+1\right)+\int\left(f_{y}(x)-f_{y}\left(b^{*}\right)\right) v(\mathrm{~d} y)=r\left(P^{*}(x)-(K-x)\right) .
$$

We see from (5.1) that $\partial_{x} P^{*}$ is continuous on $\left(b^{*}, \infty\right)$, so $f_{y} \in \mathcal{C}^{1}\left(b^{*}, \infty\right)$ and

$$
f_{y}^{\prime}(x)=\mathrm{e}^{y}\left(\partial_{x} P^{*}\left(x \mathrm{e}^{y}\right)+1\right)-\left(\partial_{x} P^{*}(x)+1\right) \geq 0
$$

if $y \geq 0$, because $x \mapsto P^{*}(x)$ is convex. So, for $x>b^{*}$ and $y>0$,

$$
f_{y}(x)-f_{y}\left(b^{*}\right) \geq 0
$$

Also, for $y \leq \ln \left(b^{*} / x\right)$, by the mean value theorem we have

$$
f_{y}(x)-f_{y}\left(b^{*}\right)=f_{y}^{\prime}(\theta)\left(x-b^{*}\right) \quad \text { for some } \theta \in\left(b^{*}, x\right),
$$


where

$$
\begin{aligned}
f_{y}^{\prime}(\theta) & =\mathrm{e}^{y}\left(\partial_{x} P^{*}\left(\theta \mathrm{e}^{y}\right)+1\right)-\left(\partial_{x} P^{*}(\theta)+1\right) \\
& \geq-\left(\partial_{x} P^{*}(\theta)+1\right) \\
& \geq-\left(\partial_{x} P^{*}(x)+1\right) .
\end{aligned}
$$

From (5.6) and (5.7) we obtain

$$
\begin{aligned}
\int_{\left\{y \leq \ln \left(b^{*} / x\right)\right\}}\left(f_{y}(x)-f_{y}\left(b^{*}\right)\right) v(\mathrm{~d} y) & \geq-\left(\partial_{x} P^{*}(x)+1\right)\left(x-b^{*}\right) v\left(\left(-\infty, \ln \left(\frac{b^{*}}{x}\right)\right)\right) \\
& =x\left(\partial_{x} P^{*}(x)+1\right) A_{x}^{b^{*}} B_{x}^{b^{*}}
\end{aligned}
$$

where

$$
A_{x}^{b^{*}}=\frac{1}{x} \frac{x-b^{*}}{\ln (x)-\ln \left(b^{*}\right)} \quad \text { and } \quad B_{x}^{b^{*}}=\ln \left(\frac{x}{b^{*}}\right) v\left(\left(-\infty, \ln \left(\frac{b^{*}}{x}\right)\right)\right) .
$$

Note that $\lim _{x \rightarrow b^{*}} A_{x}^{b^{*}}=b^{*} / x$ and $\lim _{x \rightarrow b^{*}} B_{x}^{b^{*}}=0$ (see Remark 2.1). Therefore, from (5.8) we can choose some $x_{1}>b^{*}$ such that, for $x \in\left(b^{*}, x_{1}\right)$,

$$
\int_{\left\{y \leq \ln \left(b^{*} / x\right)\right\}}\left(f_{y}(x)-f_{y}\left(b^{*}\right)\right) v(\mathrm{~d} y) \geq-\frac{x d}{4}\left(\partial_{x} P^{*}(x)+1\right) .
$$

Now, let $y \in\left(\ln \left(b^{*} / x\right), 0\right)$. Then, we have $f_{y}\left(b^{*}\right)=0$ and

$$
\begin{aligned}
f_{y}(x) & =P^{*}\left(x \mathrm{e}^{y}\right)-P^{*}(x)+x\left(\mathrm{e}^{y}-1\right) \\
& =P^{*}\left(x \mathrm{e}^{y}\right)-P^{*}(x)-x\left(\mathrm{e}^{y}-1\right) \partial_{x} P^{*}(x)+x\left(\mathrm{e}^{y}-1\right)\left(\partial_{x} P^{*}(x)+1\right) \\
& \geq x\left(\mathrm{e}^{y}-1\right)\left(\partial_{x} P^{*}(x)+1\right),
\end{aligned}
$$

since $x \mapsto P(t, x)$ is convex. We see from (2.3) that $y \mapsto \mathrm{e}^{y}-1$ is $v$-integrable, so there exists some $x_{2}>b^{*}$ such that, for $x \in\left(b^{*}, x_{2}\right)$,

$$
\int_{\left\{\ln \left(b^{*} / x\right)<y<0\right\}}\left(\mathrm{e}^{y}-1\right) v(\mathrm{~d} y) \geq-\frac{d}{4} .
$$

Therefore, from (5.10) and (5.11) we check that, for $x \in\left(b^{*}, x_{2}\right)$,

$$
\int_{\left\{\ln \left(b^{*} / x\right)<y<0\right\}}\left(f_{y}(x)-f_{y}\left(b^{*}\right)\right) v(\mathrm{~d} y) \geq-\frac{x d}{4}\left(\partial_{x} P^{*}(x)+1\right) .
$$

On the other hand, the function $f$ defined by $f(x)=P^{*}(x)-(K-x)$ is continuously differentiable, and satisfies $f\left(b^{*}\right)=0$ and $f^{\prime}(x)=\partial_{x} P^{*}(x)+1 \geq 0$. By the mean value theorem we have

$$
f(x)-f\left(b^{*}\right)=\left(\partial_{x} P^{*}(\theta)+1\right)\left(x-b^{*}\right)
$$

for some $\theta \in\left(b^{*}, x\right)$. Therefore,

$$
f(x)-f\left(b^{*}\right) \leq x\left(\partial_{x} P^{*}(x)+1\right)\left(1-\frac{b^{*}}{x}\right),
$$

since $x \mapsto P(t, x)$ is convex. So, there exists some $x_{3}>b^{*}$ such that, for $x \in\left(b^{*}, x_{3}\right)$,

$$
r\left(P^{*}(x)-(K-x)\right) \leq \frac{x d}{4}\left(\partial_{x} P^{*}(x)+1\right) .
$$


Define $x_{0}=x_{1} \wedge x_{2} \wedge x_{3}$. Recombining (5.5), (5.9), (5.12), and (5.13), we obtain, for $x \in\left(b^{*}, x_{0}\right)$,

$$
\left(x-b^{*}\right) \delta+\frac{x d}{2}\left(\partial_{x} P^{*}(x)+1\right) \leq \frac{x d}{4}\left(\partial_{x} P^{*}(x)+1\right) .
$$

This implies a contradiction since $\partial_{x} P^{*}(x)>-1$ for $x>b^{*}$.

\section{Acknowledgement}

The authors are grateful to Goran Peskir for fruitful discussions and, especially, for providing the proof of Theorem 4.1 .

\section{References}

[1] Alili, L. And Kyprianou, A. E. (2005). Some remarks on first passage of Lévy processes, the American put and pasting principles. Ann. Appl. Prob. 15, 2062-2080.

[2] Bather, J. (1970). Optimal stopping problems for Brownian motion. Adv. Appl. Prob. 2, 259-286.

[3] Bertoin, J. (1996). Lévy Processes. Cambridge University Press.

[4] Bertoin, J. (1997). Regularity of the half-line for Lévy processes. Bull. Sci. Math. 121, 345-354.

[5] Carr, P., Geman, H., Madan, D. B. and Yor, M. (2002). The fine structure of returns: an empirical investigation. J. Business 75, 305-332.

[6] Cont, R. And Tankov, P. (2004). Financial Modelling with Jump Processes. Chapman and Hall/CRC, Boca Raton, FL.

[7] El Karoui, N., Lepeltier, J.-P. and Millet, A. (1992). A probabilistic approach to the reduite in optimal stopping. Prob. Math. Statist. 13, 97-121.

[8] Kyprianou, A. E. (2006). Introductory Lectures on Fluctuations of Lévy Processes with Applications. Springer, Berlin.

[9] Lamberton, D. And Mikou, M. (2008). The critical price for the American put in an exponential Lévy model. Finance Stoch. 12, 561-581.

[10] Mikou, M. (2009). Options américaines dans le modèle exponentiel de Lévy. Doctoral Thesis, Université ParisEst.

[11] Peskir, G. (2007). Principle of smooth fit and diffusions with angles. Stochastics 79, 293-302.

[12] Peskir, G. And Shiryaev, A. (2006). Optimal Stopping and Free-Boundary Problems. Birkhäuser, Basel.

[13] Sato, K.-I. (1999). Lévy Processes and Infinitely Divisible Distributions. Cambridge University Press.

[14] Zhang, X. L. (1997). Numerical analysis of American option pricing in a jump-diffusion model. Math. Operat. Res. 22, 668-690. 